\title{
Outcome Prediction for Patients With Ischemic Stroke in Acute Care: New Three-Level Model by Eating and Bladder Functions
}

\author{
Kensalku Uchida, RPT, MS ${ }^{1}$, Yuki Uchiyama, MD, $\mathrm{PhD}^{2}$, \\ Kazuhisa Domen, $\mathrm{MD}, \mathrm{PhD}^{2}$, Tetsuo Koyama, $\mathrm{MD}, \mathrm{PhD}^{1,2}$ \\ ${ }^{1}$ Department of Rehabilitation Medicine, Nishinomiya Kyoritsu Neurosurgical Hospital, Nishinomiya; \\ ${ }^{2}$ Department of Rehabilitation Medicine, Hyogo College of Medicine, Nishinomiya, Japan
}

Objective To develop a new prediction model by combining independence in eating and bladder management functions, and to assess its utility in an acute care setting.

Methods Patients with ischemic stroke who were admitted in our acute stroke care unit $(n=250)$ were enrolled in this study. Functional Independence Measure (FIM) scores for eating and bladder management on the initial day of rehabilitative treatment (median, 3 days) were collected as predictive variables. These scores were divided into low $(<5)$ and high $(\geq 5)$ and categorized as values 0 and 1 , respectively. From the simple summation of these two-level model values, we derived a three-level model that categorized the scores as values 0,1 , and 2 . The FIMmotor scores at discharge (median, 14 days) were collected as outcome measurements. The three-level model was assessed by observing the distribution patterns of the outcome FIM-motor scores and logistic regression analyses. Results The median outcome FIM-motor score was 19 (interquartile range [IQR],13.8-45.3) for the value 0 category $(n=14), 66.5$ (IQR, 59.5-81.8) for the value 1 category $(n=16)$, and 84 (IQR, 77-89) for the value 2 category $(\mathrm{n}=95)$ in the three-level model. Data fitting by logistic regression for FIM-motor scores of 41.3 and 61.4 reached $50 \%$ probability of values 1 and 2, respectively.

Conclusion Despite the simplicity of the three-level model, it may be useful for predicting outcomes of patients with ischemic stroke in acute care.

Keywords Disability, Measurement, Prognosis, Recovery

\footnotetext{
Received October 20, 2020; Revised December 23, 2020; Accepted January 11, 2021; Published online June 14, 2021 Corresponding author: Kensaku Uchida

Department of Rehabilitation Medicine, Nishinomiya Kyoritsu Neurosurgical Hospital, 11-1 Imazu-Yamanaka-cho, Nishinomiya, Hyogo 663-8211, Japan. Tel: +81-798-33-2211, Fax: +81-798-33-2438, E-mail: ukensaku1986@gmail.com

ORCID: Kensaku Uchida (https://orcid.org/0000-0002-8447-6537); Yuki Uchiyama (https://orcid.org/0000-0002-4222-9757); Kazuhisa Domen (https://orcid.org/0000-0001-7712-7606); Tetsuo Koyama (https://orcid.org/0000-0002-1838-0684).
}

(c) This is an open-access article distributed under the terms of the Creative Commons Attribution Non-Commercial License (http://creativecommons.org/ licenses/by-nc/4.0) which permits unrestricted noncommercial use, distribution, and reproduction in any medium, provided the original work is properly cited. Copyright $\odot 2021$ by Korean Academy of Rehabilitation Medicine 


\section{INTRODUCTION}

Ischemic stroke is a major cause of disability in most advanced countries [1]. In patients with ischemic stroke, rehabilitation is often prescribed to improve activities of daily living (ADL) [2], and to maximize the efficacy of rehabilitation programs, outcome prediction is critical [3].

Various types of outcome prediction methodologies have been proposed in terms of ADL, with several studies focusing on brain imaging data $[4,5]$. In addition, magnetic resonance diffusion-tensor imaging (DTI) is one of the most promising neuroimaging techniques, although its predictive accuracy remains unclear [6]. The National Institutes of Health Stroke Scale (NIHSS) [7] was developed as another type of outcome prediction methodology that includes symptom severity and was the most commonly used methodology in previous studies $[8,9]$. Its advantage is that outcome prediction can be performed soon after stroke onset. However, there is little evidence of its predictive accuracy using early phase data (e.g., NIHSS scores within 2 days after stroke onset) [10]. Another suggested outcome prediction methodology is the use of ADL scores such as the Functional Independence Measure (FIM) and Barthel Index [11-14]. This methodology is generally more accurate than the NIHSS (clinical severity) and DTI, partially because the prediction is made within the same modality of the measurements (i.e., ADL). However, it is usually applied several weeks after stroke onset. In addition, its clinical applicability during the acute phase remains unclear.

Previous studies revealed that, inmost stroke cases, eating is the easiest and the first item to recover among ADL items $[15,16]$. This indicates that the independence of eating is essential for predicting the outcome of stroke patients during acute care. In addition, urinary incontinence is frequently observed in stroke patients [17]. A recent systematic review indicated that the independence of bladder function is a critical factor for a good outcome [18]. Mizrahi et al. [19,20] developed prediction models focusing on either eating or bladder management function within 7 days after stroke onset. They collected outcome FIM scores obtained 40-60 days after stroke onset and assessed these data in relation to dichotomous categories (dependent vs. assistance) for either eating or bladder management functions. In the present study, we attempted to extend the dichotomous categorical model by combining independence in both eating and bladder management functions and applying them to a newly developed three-level model. Our aim was to assess the clinical utility of this new three-level model.

\section{MATERIALS AND METHODS}

\section{Patients}

The work presented here is an extension of earlier studies by our research group [21] and is based on further analysis of previously reported data. The study population consisted of 250 stroke patients [21]. We retrospectively sampled patients with ischemic stroke who were admitted to the stroke care unit (SCU) of the Nishinomiya Kyoritsu Neurosurgical Hospital between April 2015 and March 2016. The study was approved by the Institutional Review Board of the Hyogo College of Medicine (No. 2454). Informed consent was obtained using the opt-out method. The diagnosis of ischemic stroke was made by examining diffusion-weighted intensity images obtained using a 3.0T magnetic resonance imaging scanner (Trio; Siemens AG, Erlangen, Germany) [22]. During hospitalization, the patients typically received conservative treatments, such as medication and rehabilitation, in accordance with guidelines of the Japanese Stroke Society $[23,24]$. According to the Japanese medical insurance system, after inpatients with stroke have received medical treatment for several weeks in an acute care hospital, they are typically discharged or transferred to a longterm rehabilitation facility [25].

From medical records during hospitalization, we collected patient data, including age, sex, modified Rankin Scale (mRS) score before stroke onset, NIHSS score, initial day of rehabilitative treatment (initial rehabilitation), length of stay (LOS), and FIM scores. Criteria for inclusion in our analytical database were pre-hospitalization $\mathrm{mRS} \leq 1$, undergoing rehabilitative treatment, and exhibiting no deterioration of symptoms (indexed by the NIHSS) within 7 days after onset. The day of admission was defined as day 1 , and the NIHSS data at initial rehabilitation were entered into the analytical database. The aim of this study was to propose a new three-level model for outcome prediction and assess its predictive accuracy. To do this, we employed two steps to evaluate the model. For the two steps of model evaluations, total samples from the analytical database were randomly al- 
located into the two groups in a 1:1 ratio (Groups 1 and 2). JMP software (version 12.0.1; SAS Institute Inc., Cary, NC, USA) was used for all statistical analyses.

\section{FIM measurements}

FIM is one of the most frequently used scoring systems for assessing ADL in the rehabilitation of stroke patients [26]. The motor component of the FIM (FIM-motor) comprises the following 13 items: eating, grooming, bathing, dressing the upper body, dressing the lower body, toileting, bladder management, bowel management, transfers to the bed/chair/wheelchair, transfers to the toilet, transfers to the bathtub/shower, walking or wheelchair propulsion, and stair climbing. FIM scores are systemically graded on a 7 -point scale: $1=$ total assistance, $2=$ maximal assistance, $3=$ moderate assistance, $4=$ minimal contact assistance, $5=$ supervision or set-up, $6=$ modified independence, and $7=$ complete independence. The total summation of FIM-motor scores (range, 13-91) is often used as an index for ADL.

In this study, FIM scores were sampled twice during hospitalization at initial rehabilitation and at discharge. The scores were assessed by physical therapists in agreement with the attending nursing staff. Data from the initial rehabilitation were used to develop prediction models by categorizing the FIM-motor scores for eating and bladder management items (see the next section). The FIM-motor score at discharge was used as an index of the final outcome. In this study, we employed FIM version 3 , which can be used for academic purposes. To ensure the reliability of the FIM data $[27,28]$, the scores were assessed by trained rehabilitation specialists, including physical therapists and occupational therapists.

\section{Prediction models}

By sampling FIM data at initial rehabilitation, we categorized eating and bladder management and developed prediction models. The cutoff point was set at 5 for the eating and bladder management items.

(1) Two-level models: FIM scores were divided into low ( $<5$ points) and high ( $\geq 5$ points). Low scores were categorized as value 0 , and high scores were categorized as value 1 . Such dichotomous two-level models were generated based on the eating and bladder management items.

(2) Three-level model: We subsequently developed a three-level model by simple summation of the assigned values ( 0 or 1$)$ in the two-level model for both the eating and bladder management items. For example, a patient with an FIM score of 3 for eating and 1 for bladder management was categorized as value 0 , another patient with an FIM score of 5 for eating and 3 for bladder management was categorized as value 1 , and yet another patient with an FIM score of 7 for eating and 5 for bladder management was categorized as value 2 .

\section{Model evaluation 1}

Categorical classifications of the prediction model scores in the two-level models were assessed using the Mann-Whitney U test with a significance level of $p<0.05$. In the three-level model, all possible pairs were assessed using Mann-Whitney $U$ test with a conservative significance level of $p<0.01$ to minimize the possibility of family wise error. Group 1 participants were sampled for these analyses. We also compared demographic data among categories of the prediction models using the MannWhitney U test.

\section{Model evaluation 2}

We further analyzed the relationships between categories of the prediction models and final FIM-motor outcomes by employing logistic regression analyses [29]. Categorical classifications of the prediction models were set as target values and final FIM-motor scores as explanatory variables. Group 2 participants were sampled for these analyses, with a significance level of $\mathrm{p}<0.05$.

The principle of logistic regression is to fit the probability $(p)$ of a dichotomous response to a linear model. The odds for these types of dichotomous responses $[p /(1-p)]$ can take any positive value. The logarithm of these odds is modeled as a simple regression:

$$
\log [p /(1-p)]=\alpha+\beta X
$$

where $\alpha$ is a constant, $\beta$ is a coefficient, and $\mathrm{X}$ is an explanatory variable.

The two-level models were assessed using simple logistic regression. Final FIM-motor scores that reached a 50\% logistic probability of value 1 were assessed for both eating and bladder management items.

To extend the utility to multilevel ordinal responses, modeling of the odds of a simple regression cumulative probability was performed at each level. For the three- 
Kensaku Uchida, et al.

Table 1. Patient characteristics

\begin{tabular}{|c|c|c|c|}
\hline & Total $(n=250)$ & Group $1(n=125)$ & Group $2(n=125)$ \\
\hline Age (yr) & $74(66-81)$ & $73(65-81)$ & $74(67-80.5)$ \\
\hline \multicolumn{4}{|l|}{ Sex } \\
\hline Male & 155 & 74 & 81 \\
\hline Female & 95 & 51 & 44 \\
\hline NIHSS & $3(1-6)$ & $3(1.5-6)$ & $3(1-5)$ \\
\hline Initial rehabilitation (day) & $3(2-3)$ & $2(2-3)$ & $3(2-3)$ \\
\hline \multicolumn{4}{|l|}{ FIM eating score } \\
\hline 1 & 27 & 15 & 12 \\
\hline 2 & 1 & 0 & 1 \\
\hline 3 & 3 & 1 & 2 \\
\hline 4 & 5 & 3 & 2 \\
\hline 5 & 13 & 6 & 7 \\
\hline 6 & 83 & 40 & 43 \\
\hline 7 & 118 & 60 & 58 \\
\hline \multicolumn{4}{|c|}{ FIM bladder management score } \\
\hline 1 & 46 & 21 & 25 \\
\hline 2 & 5 & 1 & 4 \\
\hline 3 & 3 & 1 & 2 \\
\hline 4 & 3 & 2 & 1 \\
\hline 5 & 8 & 6 & 2 \\
\hline 6 & 5 & 2 & 3 \\
\hline 7 & 180 & 92 & 88 \\
\hline LOS (day) & $14(10-24)$ & $14(10-24)$ & $14(10-24.5)$ \\
\hline FIM-motor at discharge & $83(68-88)$ & $82(68-88)$ & $83(67-88.5)$ \\
\hline
\end{tabular}

Values are presented as median (interquartile range).

For two-step procedures of model evaluation, total samples from the analytical database were randomly allocated into one of two groups in a 1:1 ratio (Groups 1 and 2).

NIHSS, National Institutes of Health Stroke Scale; FIM, Functional Independence Measure; LOS, length of stay.

(A)

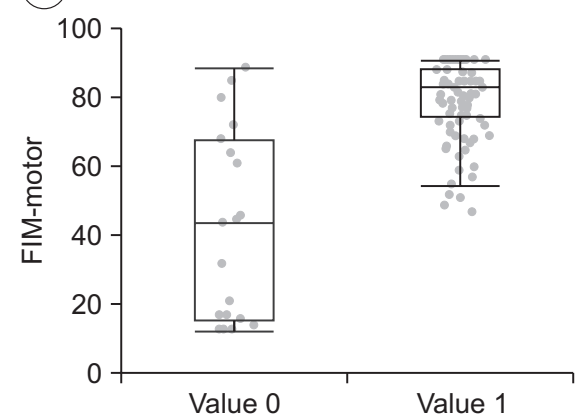

(B)

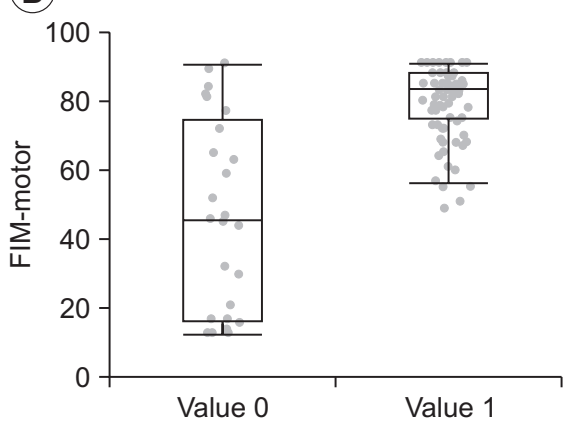

(C)

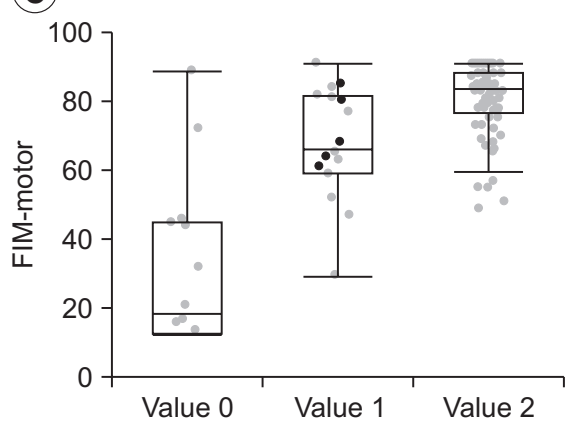

Fig. 1. Box charts and plots for data distributions of the prediction models derived from Group 1 samples: (A) twolevel model eating, (B) two-level model bladder management, and (C) three-level model. Refer to Table 2 for statistical comparisons. Black dots in the value 1 category for the three-level model represent patients who were assigned value 1 in the two-level model bladder management and value 0 in the two-level model eating. FIM, Functional Independence Measure. 
level model responses ( $p 1, p 2$, and $p 3$; sum equals 1 ), the logarithm of the odds was modeled as two simple regressions for the three-level model responses (note that single $\beta$ and two levels of $\alpha$ were assessed).

$$
\begin{aligned}
& \log [p 1 /(p 2+p 3)]=\alpha_{1}+\beta_{1} X \\
& \log [(p 1+p 2) / p 3]=\alpha_{2}+\beta_{1} X
\end{aligned}
$$

The three-level model was assessed using a multilevel logistic regression. Final FIM-motor scores that reached $50 \%$ logistic probability of values 1 and 2 were also assessed.

\section{RESULTS}

\section{Patient profiles}

A total of 527 patients with ischemic stroke were admitted to the SCU during the study period. Of these, 425 received rehabilitative treatment. Patients with an NIHSS score of 0 and a threat to life due to severe stroke typically did not receive rehabilitative treatment. We excluded 175 patients from our analytical database because they did not meet the pre-hospitalization mRS criteria $(n=154)$, exhibited deterioration of symptoms within 1 week after onset $(n=17)$, or were absent from data collection $(n=4)$. Subsequently, 250 patients were included in the final database.

Table 1 shows patient demographics from our analytical database. The median age was 74 years, and there were more men than women $(62 \% ; n=155 / 250)$. The median of the NIHSS score at initial rehabilitation was 3 (interquartile range [IQR], 1-6). The median at initial rehabilitation was 3 (IQR, 2-3). Median LOS was 14 days. The median FIM-motor score at discharge was 83 (IQR, 68-88).

\section{Model evaluation 1}

Fig. 1 and Table 2 show the categorical classifications of the prediction models. In the two-level models, analyses detected statistically significant differences in FIM-motor scores at discharge for both eating and bladder management items. For eating, the median FIM-motor score was 44 (IQR, 16-68) in the value 0 category and 83.5 (IQR, 75-89) in the value 1 category. For bladder management, the median FIM-motor score was 46 (IQR, 17-74.5) in the value 0 category and 84 (IQR, 75.5-89) in the value 1 category. Notably, the data distribution of the value 0 cat-

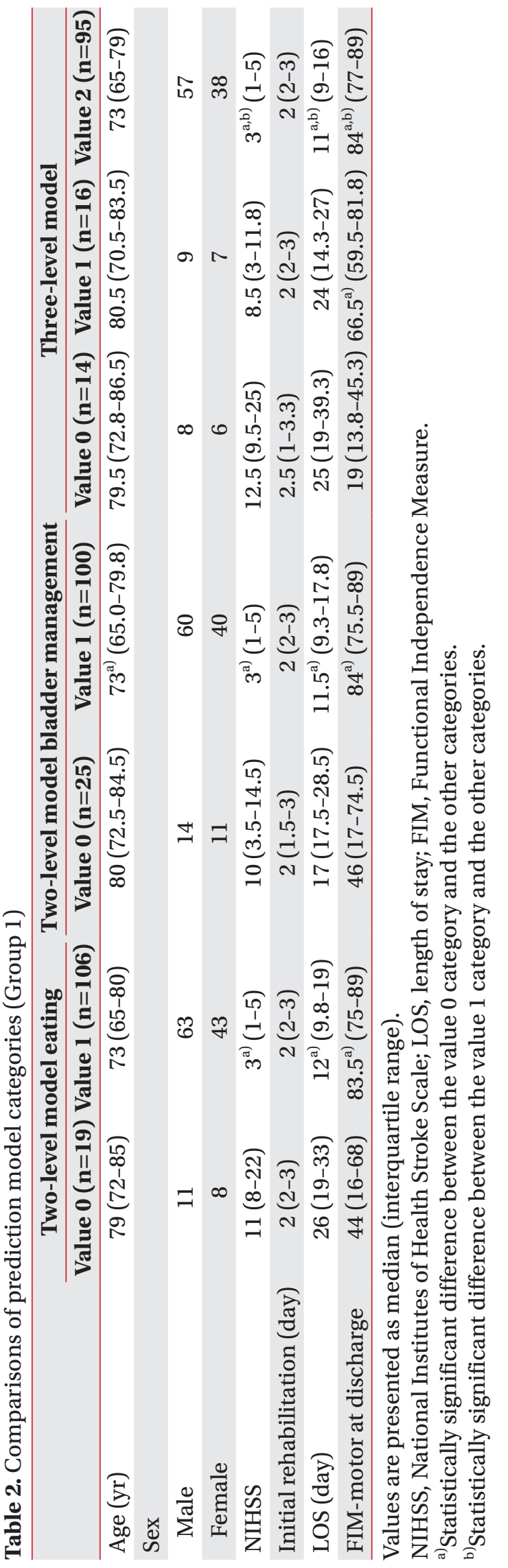

www.e-arm.org 

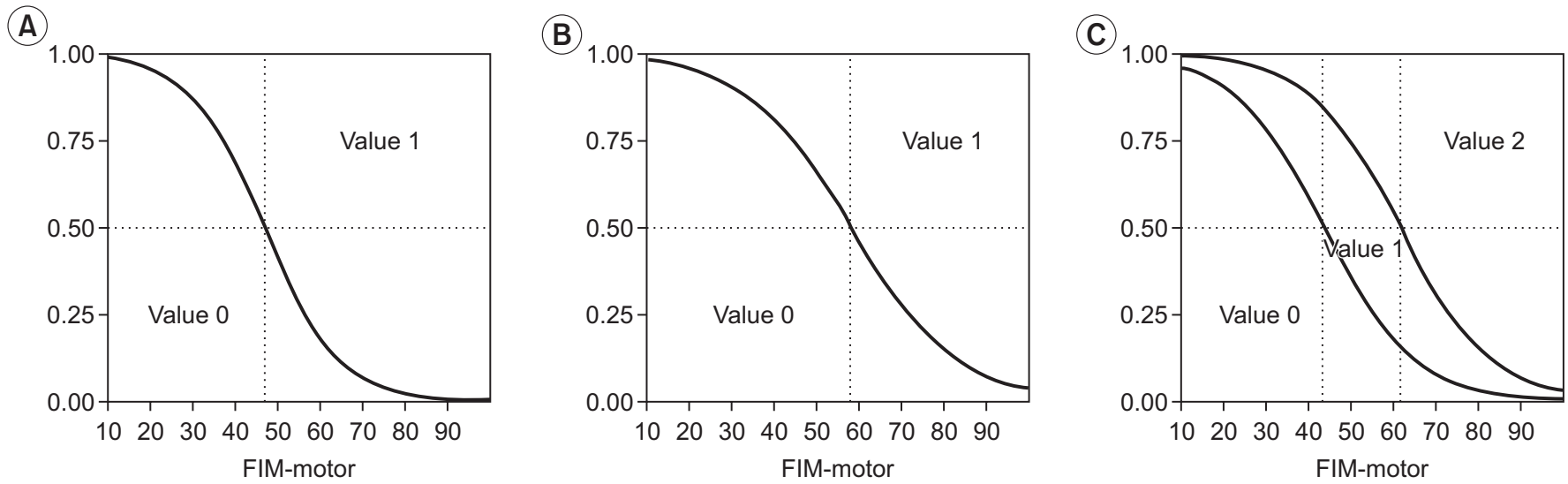

Fig. 2. Logistic probability curves for the relationship between the categories of the prediction models and FIM-motor scores at discharge: (A) two-level model eating, (B) two-level model bladder management, and (C) three-level model. Vertical axes indicate logistic probability and horizontal axes show FIM-motor scores at discharge. (A, B) The distance from the curve to the top of the graph is the probability of the value 1 category. (C) The left curve defines the probability for the value 1 category, and the right curve defines the probability for the value 2 category. FIM, Functional Independence Measure.

egory was much larger than that of the value 1 category. Such observation was common in two-level models for eating and bladder management.

In line with the findings from the two-level models, analyses for the three-level model detected statistically significant differences in FIM-motor scores at discharge in all possible pairs. However, the patterns of the data distributions were different from those of the two-level models. Median FIM-motor score was 19 (IQR, 13.8-45.3) for the value 0 category, 66.5 (IQR, 59.5-81.8) for the value 1 category, and 84 (IQR, 77-89) for the value 2 category; the data were evenly distributed in the FIM-motor range among these three categories. The value 1 category comprised 16 patients (Table 2). Of these, 11 were from the value 1 category in the two-level eating model, and the remaining 5 were from the value 1 category in the two-level bladder management model. This observation indicated the dominance of the eating items in the value 1 category for the three-level model.

\section{Model evaluation 2}

Fig. 2 shows the logistic probability curves for the categories of all models in relation to the FIM-motor scores. Table 3 presents the parameter estimates of the logistic regression analyses. These results show that the relationships between the categories in the prediction models and the FIM-motor scores sufficiently fit the logistic probability. In the two-level model for eating, an FIM-
Table 3. Parameter estimates for multilevel logistic regression analyses between categories and FIM-motor scores in prediction models (Group 2)

\begin{tabular}{|c|c|c|c|c|}
\hline & \multicolumn{4}{|c|}{ Ordinal logistics } \\
\hline & $\beta$ & $\alpha$ (score 0 & 1) $\alpha$ (score 1-2) & $\mathbf{R}^{2}$ \\
\hline \multicolumn{5}{|c|}{ Two-level model eating } \\
\hline Estimates & -0.115 & 5.372 & - & 0.571 \\
\hline SE & 0.025 & 1.491 & - & - \\
\hline p-value & $<0.001$ & $<0.001$ & - & $<0.001$ \\
\hline
\end{tabular}

Two-level model bladder management

\begin{tabular}{|c|c|c|c|c|}
\hline Estimates & -0.081 & 4.691 & - & 0.315 \\
\hline SE & 0.016 & 1.189 & - & - \\
\hline p-value & $<0.001$ & $<0.001$ & - & $<0.001$ \\
\hline \multicolumn{4}{|c|}{ Three-level model } & - \\
\hline Estimates & -0.094 & 4.096 & 5.805 & 0.334 \\
\hline SE & 0.016 & 0.994 & 1.108 & - \\
\hline $\mathrm{p}$-value & $<0.001$ & $<0.001$ & $<0.001$ & $<0.001$ \\
\hline
\end{tabular}

FIM, Functional Independence Measure; SE, standard error.

motor score of 46.7 reached the $50 \%$ probability of a value of 1 . However, for bladder management, the score was 58.1. In the three-level model, the left curve defines the probability for the value 1 category, and the right curve defines the probability for the value 2 category. An FIMmotor score of 41.3 and 61.4 reached $50 \%$ probability of values 1 and 2 , respectively. 


\section{DISCUSSION}

We developed a three-level model in an attempt to extend the previously reported two-level categorical models for outcome prediction based on eating and bladder management $[19,20]$. Although the three-level model is a simple summation of the two-level model, its advantages over the two-level model include: (1) equal categorization of the FIM-motor score distribution at discharge, and (2) the lower and upper FIM-motor scores of the three categories were approximately 40 and 60 , respectively, which is meaningful in terms of clinical utility.

Predicting outcomes soon after stroke onset is critical for scheduling appropriate rehabilitative treatments. NIHSS is frequently used for predicting outcomes $[8,9]$, but it assesses symptoms of neural deficits, rather than functional independence in ADL. FIM is an assessment tool for ADL and is widely used in various medical and social welfare settings, including acute medical care. Some FIM items are accessible, while others are difficult to assess in an acute medical care setting. Eating and bladder management are easy to assess even during the super-acute phase; eating usually takes place 3 times daily during meal times, while urination takes place approximately 5-10 times daily. In contrast, transfer to the bathtub/ shower and stair climbing are difficult to assess because acute medical settings such as the SCU in the present study are not equipped with such facilities. Thus, the two FIM items, eating and bladder management, are commonly easy to assess in most acute medical care settings, including the SCU.

In this study, we employed a cutoff value of 5 points (supervision) for the FIM data, while Mizrahi et al. set a cutoff value of 6 points (modified independence level) $[19,20]$. In this study, we employed a cutoff point as the supervision level because the data sampled in our SCU were nearly always in the context of nurses attending to patients. In addition, our research team has been conducting a series of studies to investigate the psychometric properties of FIM in stroke patients $[21,29]$. The studies that employed a cutoff value of 5 points were successful in outlining the psychometric properties of the FIM. Accordingly, we kept our cutoff point the same as in our previous studies.

As Fig. 1 shows, the three-level model classified outcome FIM-motor scores are in a relatively even distribu- tion pattern. To further investigate the borders of these three categories, we applied an ordinal logistic regression analysis. Fig. 2 shows that FIM-motor scores of 40 and 60 reached $50 \%$ probability of values 1 and 2, respectively, and Koyama et al. [29] defined item difficulty of single FIM items relative to the summation of FIM-motor scores in patients after stroke. They reported that the supervision level was reached when the FIM-motor scores were approximately 50 in terms of the ability to transfer to the bed/chair/wheelchair. In line with the report by Kwon et al. [30], the authors assessed the relationship between mRS and FIM-motor scores. They reported that the probability of gait independence ( $\mathrm{mRS} \leq 3$ ) exceeded $50 \%$ when the FIM-motor scores reached 65 or higher. Since both transfers to the bed/chair/wheelchair and gait are important indices in ADL, our three-level model offers good benchmarks for stroke rehabilitation.

Most previous studies sampled outcome data at 3-6 months after stroke [11,12]. Mizrahi et al. [19,20] sampled outcome scores during an earlier phase, i.e., 40-60 days after stroke. However, we sampled outcome scores 2-4 weeks after stroke onset in the present study, which is much earlier than in previous studies. We defined outcomes according to this early period because the average LOS in acute care hospitals in 2017 was approximately 16 days in Japan [31], which is consistent with the present study. In Japan, the medical insurance system is government-controlled; therefore, hospital staff and patients need to decide the setting of the early phase of stroke treatment, i.e., transfer to rehabilitation facilities or discharge directly home [32]. Despite the early outcome sampling period in the present study, our methodology matches the realistic needs of stroke rehabilitation during acute care in Japan.

In this study, we developed a three-level model by simple summation of dichotomous classifications of FIM levels for eating and bladder management. This simple summation methodology implies that the weights for eating and bladder management are equal for outcome prediction. To further investigate the weights for these two items, we used data from Group 1 to perform preliminary multivariate regression analysis where the values ( 0 or 1$)$ for eating and bladder management were set as independent variables, and FIM-motor scores at discharge were set as the target values. Estimated regression coefficients were 23.5 for eating and 21.5 for bladder management, 
and the intercept was 37.0; the ratio of the two coefficients was nearly $1: 1$. Given these preliminary findings, we employed a simple summation, i.e., the three-level model.

This study has several limitations. First, samples were collected from an acute care hospital. Accordingly, outcome assessments were obtained approximately 2-4 weeks after stroke onset, and in contrast, most stroke outcome studies sampled long-term care several months after stroke. Thus, the terminology "outcome" in the present study requires attention. Second, to minimize variability, we sampled data from patients who were independent in their ADL before stroke. Subsequently, we excluded patients with commonly observed geriatric comorbidities (e.g., dementia). Thus, we excluded nearly one-third of the total samples from our final analytical database. Therefore, careful consideration should be taken when applying the three-level model to the general population. Third, we sampled data regardless of lesion site, even though clinical manifestations of brainstem lesions (e.g., bulbar palsy) are different from those of supratentorial lesions (e.g., pseudo-bulbar palsy). However, the present results suggest that our three-level model is useful for clinicians who need to determine whether patients should be transferred to a rehabilitation facility or discharged home during the early phase of stroke treatment.

\section{CONFLICT OF INTEREST}

No potential conflict of interest relevant to this article was reported.

\section{ACKNOWLEDGMENTS}

This study was partially supported by a Grant-in-Aid for Scientific Research (B), Japan Society for the Promotion of Science (JSPS KAKENHI [JP16H03209]).

\section{AUTHOR CONTRIBUTION}

Conceptualization: Uchida K, Domen K. Data collection: Uchida K. Methodology: Uchida K, Koyama T. Formal analysis: Uchida K, Koyama T. Funding acquisition: Koyama T. Project administration: Uchiyama Y, Domen K, Koyama T. Visualization: Uchida K, Uchiyama Y, Koyama
T. Writing-original draft: Uchida K, Uchiyama Y, Koyama

T. Writing-review and editing: Uchida K, Koyama T. Approval of final manuscript: all authors.

\section{REFERENCES}

1. Miller EL, Murray L, Richards L, Zorowitz RD, Bakas T, Clark P, et al. Comprehensive overview of nursing and interdisciplinary rehabilitation care of the stroke patient: a scientific statement from the American Heart Association. Stroke 2010;41:2402-48.

2. Langhorne P, Bernhardt J, Kwakkel G. Stroke rehabilitation. Lancet 2011;377:1693-702.

3. Kwakkel G, Kollen BJ. Predicting activities after stroke: what is clinically relevant? Int J Stroke 2013;8:25-32.

4. Kim B, Winstein C. Can neurological biomarkers of brain impairment be used to predict poststroke motor recovery? A systematic review. Neurorehabil Neural Repair 2017;31:3-24.

5. Sagnier S, Sibon I. The new insights into human brain imaging after stroke. J Neurosci Res 2019 Sep 9 [Epub]. https://doi.org/10.1002/jnr.24525.

6. Moura LM, Luccas R, de Paiva JP, Amaro E Jr, Leemans A, Leite CD, et al. Diffusion tensor imaging biomarkers to predict motor outcomes in stroke: a narrative review. Front Neurol 2019;10:445.

7. Brott T, Adams HP Jr, Olinger CP, Marler JR, Barsan WG, Biller J, et al. Measurements of acute cerebral infarction: a clinical examination scale. Stroke 1989;20:864-70.

8. Lai SM, Duncan PW, Keighley J. Prediction of functional outcome after stroke: comparison of the Orpington Prognostic Scale and the NIH Stroke Scale. Stroke 1998;29:1838-42.

9. Saito J, Koyama T, Domen K. Long-term outcomes of FIM motor items predicted from acute stage NIHSS of patients with middle cerebral artery infarct. Ann Rehabil Med 2018;42:670-81.

10. Kwakkel G, Veerbeek JM, van Wegen EE, Nijland R, Harmeling-van der Wel BC, Dippel DW. Predictive value of the NIHSS for ADL outcome after ischemic hemispheric stroke: does timing of early assessment matter? J Neurol Sci 2010;294:57-61.

11. Koyama T, Matsumoto K, Okuno T, Domen K. A new method for predicting functional recovery of stroke patients with hemiplegia: logarithmic modelling. Clin 
Rehabil 2005;19:779-89.

12. Sonoda S, Saitoh E, Nagai S, Okuyama Y, Suzuki T, Suzuki M. Stroke outcome prediction using reciprocal number of initial activities of daily living status. J Stroke Cerebrovasc Dis 2005;14:8-11.

13. De Wit L, Putman K, Devos H, Brinkmann N, Dejaeger E, De Weerdt W, et al. Long-term prediction of functional outcome after stroke using single items of the Barthel Index at discharge from rehabilitation centre. Disabil Rehabil 2014;36:353-8.

14. Brown AW, Therneau TM, Schultz BA, Niewczyk PM, Granger CV. Measure of functional independence dominates discharge outcome prediction after inpatient rehabilitation for stroke. Stroke 2015;46:1038-44.

15. Granger CV, Hamilton BB, Linacre JM, Heinemann AW, Wright BD. Performance profiles of the functional independence measure. Am J Phys Med Rehabil 1993;72:84-9.

16. Stineman MG, Fiedler RC, Granger CV, Maislin G. Functional task benchmarks for stroke rehabilitation. Arch Phys Med Rehabil 1998;79:497-504.

17. Brittain KR, Peet SM, Castleden CM. Stroke and incontinence. Stroke 1998;29:524-8.

18. van Almenkerk S, Smalbrugge M, Depla MF, Eefsting JA, Hertogh CM. What predicts a poor outcome in older stroke survivors? A systematic review of the literature. Disabil Rehabil 2013;35:1774-82.

19. Mizrahi EH, Arad M, Weiss A, Leibovitz A, Adunsky A. Eating management and functional outcome of elderly patients with symptomatic ischemic stroke undergoing inpatient rehabilitation. Geriatr Gerontol Int 2013;13:701-5.

20. Mizrahi EH, Waitzman A, Arad M, Blumstein T, Adunksy A. Bladder management and the functional outcome of elderly ischemic stroke patients. Arch Gerontol Geriatr 2011;53:e125-8.

21. Uchida K, Uchiyama Y, Domen K, Koyama T. Item difficulties of the FIM-motor subscale in patients with ischemic stroke during acute care: an ordinal logistic modeling study. Prog Rehabil Med 2020;5:20200022.

22. Koyama T, Domen K. Diffusion tensor fractional anisotropy in the superior longitudinal fasciculus correlates with Functional Independence Measure cognition scores in patients with cerebral infarction. J Stroke Cerebrovasc Dis 2017;26:1704-11.
23. Shinohara Y, Yanagihara T, Abe K, Yoshimine T, Fujinaka T, Chuma T, et al. VII. Rehabilitation. J Stroke Cerebrovasc Dis 2011;20(4 Suppl):S145-80.

24. Shinohara Y, Yanagihara T, Abe K, Yoshimine T, Fujinaka T, Chuma T, et al. II. Cerebral infarction/transient ischemic attack (TIA). J Stroke Cerebrovasc Dis 2011;20(4 Suppl):S31-73.

25. Miyai I, Sonoda S, Nagai S, Takayama Y, Inoue Y, Kakehi A, et al. Results of new policies for inpatient rehabilitation coverage in Japan. Neurorehabil Neural Repair 2011;25:540-7.

26. Linacre JM, Heinemann AW, Wright BD, Granger CV, Hamilton BB. The structure and stability of the Functional Independence Measure. Arch Phys Med Rehabil 1994;75:127-32.

27. Hamilton BB, Laughlin JA, Fiedler RC, Granger CV. Interrater reliability of the 7-level functional independence measure (FIM). Scand J Rehabil Med 1994;26:115-9.

28. Ottenbacher KJ, Hsu Y, Granger CV, Fiedler RC. The reliability of the functional independence measure: a quantitative review. Arch Phys Med Rehabil 1996;77:1226-32.

29. Koyama T, Matsumoto K, Okuno T, Domen K. Relationships between independence level of single motor-FIM items and FIM-motor scores in patients with hemiplegia after stroke: an ordinal logistic modelling study. J Rehabil Med 2006;38:280-6.

30. Kwon S, Hartzema AG, Duncan PW, Min-Lai S. Disability measures in stroke: relationship among the Barthel Index, the Functional Independence Measure, and the Modified Rankin Scale. Stroke 2004;35:918-23.

31. Organisation for Economic Co-operation and Development. OECD data: length of hospital stay [Internet]. Paris, France: Organisation for Economic Co-operation and Development; c2021 [cited 2021 May 18]. Available from: https://data.oecd.org/healthcare/ length-of-hospital-stay.htm.

32. Ministry of Health, Labour and Welfare. Annual Health, Labour and Welfare Report 2016: health and medical services [Internet]. Tokyo, Japan: Ministry of Health, Labour and Welfare; c2021 [cited 2021 May 18]. Available from: https://www.mhlw.go.jp/english/ wp/wp-hw10/dl/02e.pdf. 